\title{
Klinefelter syndrome and testosterone treatment: a national cohort study on thrombosis risk
}

\author{
Simon Chang ${ }^{1,2}$, Christian Fynbo Christiansen ${ }^{3}$, Anders Bojesen ${ }^{4}$, Svend Juul ${ }^{5}$, Anna-Marie B Münster ${ }^{1}$ and \\ Claus H Gravholt ${ }^{2,6}$ \\ ${ }^{1}$ Unit for Thrombosis Research, Institute of Regional Health Research, University of Southern Denmark and Department of Clinical Biochemistry, Hospital \\ of South West Denmark, Esbjerg, Denmark \\ ${ }^{2}$ Department of Endocrinology and Internal Medicine, Aarhus University Hospital, Aarhus N, Denmark \\ ${ }^{3}$ Department of Clinical Epidemiology, Aarhus University Hospital, Aarhus N, Denmark \\ ${ }^{4}$ Department of Clinical Genetics, Aarhus University Hospital, Aarhus N, Denmark \\ ${ }^{5}$ Department of Public Health, Aarhus University, Aarhus C, Denmark \\ ${ }^{6}$ Department of Molecular Medicine, Aarhus University Hospital, Aarhus N, Denmark
}

Correspondence should be addressed to S Chang: simon.chang@rsyd.dk

\begin{abstract}
Objectives: Klinefelter syndrome (KS), 47,XXY, can be viewed as a disease model for investigating the risk of thrombosis in male hypogonadism and the subsequent risk related to testosterone treatment. We describe rates of thrombotic risk factors, thrombosis and thrombosis mortality in KS and the association with testosterone treatment.

Methods: National registry-based matched cohort study with follow-up from 1995 to 2016 set in Denmark. For the study, 1155 men with KS were each matched by year and month of birth to 100 men from the background population. First thrombotic events and thrombosis mortality was evaluated by event rates and hazard ratios (HRs) and by applying testosterone treatment as a time-dependent covariate.

Results: The KS cohort had higher incidence of venous thromboembolism relative to the comparison cohort $(\mathrm{HR}, 3.95 ; 95 \% \mathrm{Cl}, 2.83-5.52)$. Total thrombotic deaths were increased in $\mathrm{KS}(\mathrm{HR}, 1.76 ; 95 \% \mathrm{Cl}, 1.18-2.62)$, and all-cause mortality was increased in KS following arterial thrombosis (HR 1.73; 95\% Cl 1.22-2.47). Only $48.7 \%$ of men with KS redeemed prescriptions for testosterone. Untreated men with KS were on average born 12 years before those treated, and the majority of untreated men with KS with available biochemistry were hypogonadal. Testosterone treatment in KS was associated with a non-significant decrease in venous thromboembolism and thrombotic deaths. Conclusion: Thrombosis and thrombotic deaths are increased in KS. Only half of the men with $\mathrm{KS}$ ever received testosterone treatment, despite overt hypogonadism in the nontreated. Testosterone treatment in Klinefelter syndrome was insignificantly associated with lower incidence rates of venous thrombosis and thrombotic deaths.
\end{abstract}

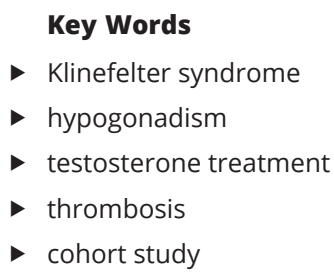

Endocrine Connections (2020) 9, 34-43

\section{Introduction}

Men with 47,XXY Klinefelter syndrome (KS) commonly present hypergonadotropic hypogonadism and are commonly treated with testosterone supplementation therapy (1). However, this treatment is almost entirely based on our knowledge about normal physiology, and very little research has investigated the key aspects of 
testosterone supplementation therapy in KS (1), including whether the treatment could have unwarranted effects. The practice of evidence-based medicine is the foundation for proficient care of any condition. For rare conditions, obtaining clear empirical evidence can be challenging especially due to difficulties in the recruitment of a high enough number of patients and less interest from funding sources for supporting the research. Both male hypogonadism and testosterone treatment in men have been associated with an increased risk of thrombotic events $(2,3,4,5,6,7)$, and men with KS exhibit an up to six-fold increased risk of venous thromboembolisms (VTE) $(8,9)$, increased risk of arterial thrombosis (ATE) (8), and a more than two-fold increased rate of mortality related to thrombotic events $(10,11,12)$. Thrombotic risk in men with KS could be affected directly by the presence of an extra X chromosome, subsequent hypogonadism, and the induction of a vicious cycle of truncal obesity and insulin insensitivity $(1,13)$. This unfavourable metabolism then leads to increased risk of disease entities associated with cardiovascular risk and furthermore skewing of the haemostatic balance (14) and increased platelet aggregation (15). Testosterone treatment could reduce thrombotic risk by counteracting the vicious metabolic cycle present in KS (16). Contrary, it could also be speculated that testosterone treatment would increase thrombotic risk by, for instance, inducing erythrocytosis $(17,18)$.

We performed a nationwide cohort study using men in the background population as a comparison cohort and integrating data regarding diagnoses, medication use, and clinical biochemistry from national registries. Our aim was to describe rates of thrombosis and thrombotic risk factors and, for the first time in KS using a national prescription registry, provide an epidemiological assessment of testosterone treatment in KS and specifically the relation between testosterone treatment and thrombotic risk among men with KS.

\section{Materials and methods}

\section{Setting and data sources}

This was a registry-based national matched cohort study. The Danish health system provides free access to general practice and hospitals as well as partial reimbursement of prescription medicine. All contacts with the healthcare system are registered using the individually assigned Civil
Registration Number (19), permitting linkage of highquality data. The study was approved by The Danish Data Protection Agency (record number: 1-16-02-568-15).

Hospital discharge diagnoses were retrieved from The Danish National Patient Registry (20) according to the International Classification of Diseases (ICD) eighth or tenth revision. A complete list of ICD codes applied is provided in the Supplementary Table 1 (see section on supplementary materials given at the end of this article). Deaths and date of death were retrieved from the Civil Registration System (19). Cause of death was retrieved from The Danish Registry of Causes of Death (21) according to the ICD-10 classification. Medications were identified according to Anatomical Therapeutic Chemical classification (ATC) codes, and prescription history was obtained from the Danish National Prescription Registry (22). A detailed list of all ATC codes applied is provided in the Supplementary Table 2. Results from biochemical analyses were retrieved from central databases containing all biochemical analyses performed at hospital laboratories in each of the five administrative Regions of Denmark. In the Region of Southern Denmark and the North Denmark Region laboratory results are available from 1992, in the Central Denmark Region from 2008, in the Region Zealand from 2011, and in the Capital Region of Denmark from 1988 until now.

\section{Population}

KS was defined as any 47,XXY, 47, XXY/46, XY mosaic or other $47, \mathrm{XXY}$ mosaic karyotype, and cases were identified from the Danish Central Cytogenetic Register (23). Karyotypes with more than one supernumerary $\mathrm{X}$ chromosome were not included, as these are now considered as separate conditions (1). Each KS case was matched with up to 100 men in the background population by year and month of birth.

\section{Study period and exposure time}

To ensure at least 1 year of prescription data for all participants, the study period chosen was from 1995 through 2016. All participants were considered at risk from birth, since KS is a congenital condition, and entered the study on 1 January 1995 or the date of birth, whichever came last. Censoring was at the time of the death, or emigration, or on 31 December 2016, whichever came first. https://ec.bioscientifica.com

https://doi.org/10.1530/EC-19-0433 (c) 2020 The authors Published by Bioscientifica Ltd

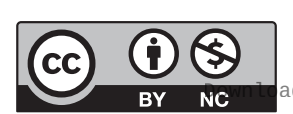

This work is licensed under a Creative Commons Attribution-NonCommercial 4.0 International License. ded from Bioscientifica.com at 04/26/2023 06:36:18AM via free access 


\section{Evaluated outcomes}

VTE was defined as the composite outcome of deep venous thrombosis, pulmonary embolism, cerebral vein thrombosis, or portal vein thrombosis. A diagnosis of first time in- or outpatient VTE was considered valid if followed by a redeemed prescription for anticoagulation within 30 days (24). The positive predictive value for this algorithm in the identification of VTE has been estimated to be 90\% (24). To not exclude those dying before redeeming a prescription, any first VTE diagnosis followed by death within 30 days was also considered valid. ATE was defined as the composite of myocardial infarction, stroke, or transient ischemic attack. The positive predictive value of myocardial infarction and stroke diagnoses among men in The Danish National Patient Registry is above $95 \%$ and around $60 \%$ for transient ischemic attack (20, 25). First time ATE events were evaluated in a similar manner as VTE, demanding a redeemed prescription of anti-platelet medication or death within 30 days. Diagnoses of atrial arrhythmia (fibrillation or flutter), angina pectoris, arteriosclerosis, hypertension, diabetes, and hypercholesterolemia, all co-morbidities associated with thrombosis risk $(26,27)$, were also identified in The Danish National Patient Registry going back to 1977 when the registry was created.

Death due to thrombosis was defined as any VTE or ATE diagnosis and registered as either primary or auxiliary cause of death.

For all treatments, the day of first redeemed prescription was considered as the onset of treatment. Use of disease-specific medications were assessed to indirectly evaluate the incidence and timing of comorbidities, for example, insulin or oral antidiabetics as an indicator for diabetes and statins for hypercholesterolemia.

Results from clinical biochemistry were assessed to evaluate the presence of hypogonadism in untreated KS. The data hold no information about the applied assay methodology and therefore testosterone levels below the given reference range or luteinizing hormone levels two-fold above the upper reference limit were considered indicative of hypogonadism.

Blood levels of haemoglobin, haematocrit, and testosterone within \pm 90 days of the thrombotic events were assessed to evaluate the presence of erythrocytosis in treated KS. Abnormal test results were defined as values outside the given reference range, as the methodology varies between laboratories and over time.

\section{Statistical analysis}

For all VTE, ATE, and mortality outcomes, we computed hazard ratios (HR) with robust variance estimates using stratified Cox regression. Each stratum comprised one KS case and the matched individuals in the comparison cohort. Only the first diagnosis was considered. Adjustment, using medication as a proxy for co-morbidity, was applied in a separate analysis. For this, use of antidiabetics, antihypertensives, statins, anticoagulation, platelet inhibitors, and anticoagulation were added as time-varying covariates with exposure starting at first redeemed prescription. Similarly, we computed HR's for first prescriptions for selected medications or incidence of co-morbidity diagnoses. To evaluate mortality following VTE or ATE, a separate analysis was carried out including only participants with thrombosis and with follow-up starting at the time of thrombosis. To evaluate effects on various outcomes of testosterone treatment, we conducted a consecutive set of Cox analyses within the KS population, applying testosterone treatment as a time varying covariate and adjusting for year of birth or year of birth and use of medications as described previously. Contingency of categorical variables were evaluated applying Fisher's exact test. Analyses were performed using Stata 15.1 (StataCorp, College Station).

\section{Results}

\section{Study population and thrombotic events}

A total of 1155 men with KS were identified (Table 1). The comparison cohort comprised 111,765 individuals. The mean age at entry for both groups was just over 25 years, and the majority (78.8\%) of participants had data available for the entire study period (22 years, Table 1 ).

In the KS cohort, 17.0 (95\% CI, 12.4-23.4) VTE events were recorded per 10,000 person-years and in the comparison cohort, 4.3 (4.0-4.6) VTE events were recorded per 10,000 person-years (Table 1), leading to a four-fold increased VTE incidence among males with KS compared with the comparison cohort (Fig. 1A and Table 1).

In the KS cohort, 32.0 (95\% CI, 25.4-40.4) ATE events were recorded per 10,000 person-years. In the comparison cohort, 30.0 (29.3-30.8) ATE events were recorded per 10,000 person-years (Table 1). The incidence of ATE was not different between men with KS and the comparison cohort (Fig. 1B and Table 1).
This work is licensed under a Creative Commons Attribution-NonCommercial 4.0 International License. ded from Bioscientifica.com at 04/26/2023 06:36:18AM via free access 
Table 1 Participant characteristics, thrombotic events and thrombotic deaths.

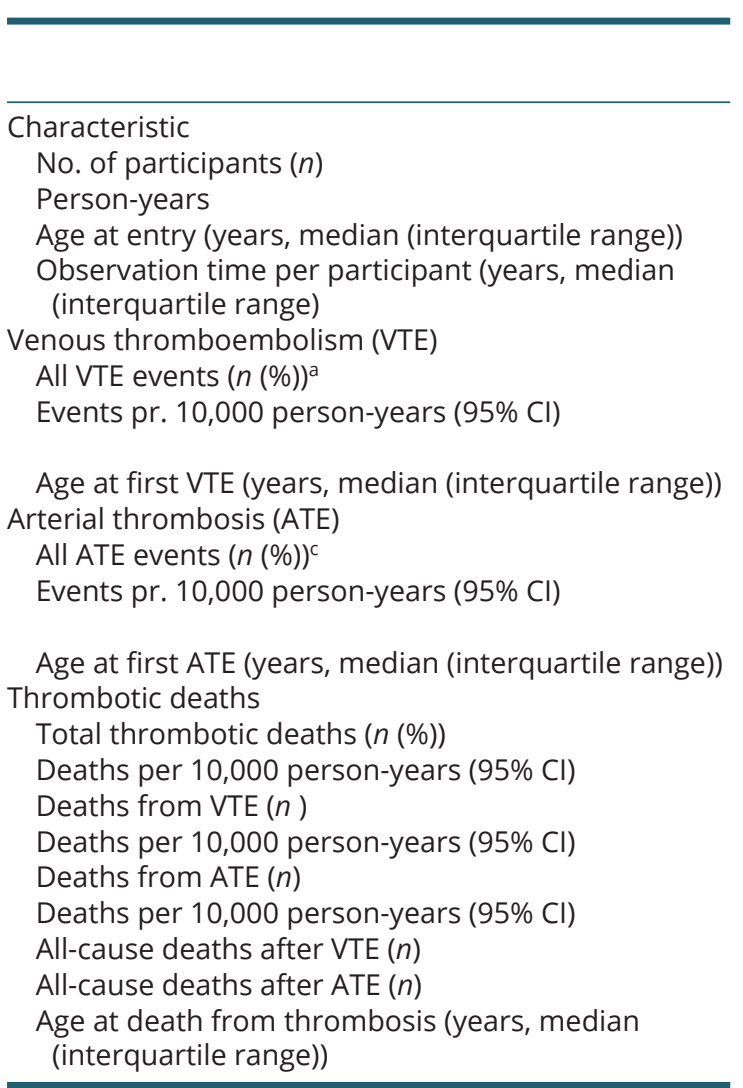

\begin{tabular}{|c|c|c|}
\hline KS & Comparison cohort & $\begin{array}{l}\text { HR }(95 \% \mathrm{Cl}) \text { : KS vs the } \\
\text { comparison cohort }\end{array}$ \\
\hline 1,155 & 111,765 & - \\
\hline 22,616 & $2,253,137$ & \\
\hline $25.8(12.1-41.5)$ & $25.2(11.9-40.1)$ & - \\
\hline $22.0(22.0-22.0)$ & $22.0(22.0-22.0)$ & - \\
\hline $38(3.3)$ & 964 (0.9) & - \\
\hline $17.0(12.4-23.4)$ & $4.3(4.0-4.6)$ & $\begin{array}{l}3.95(2.83-5.52) \\
3.11(2.18-4.45)^{b}\end{array}$ \\
\hline $50.6(41.1-62.1)$ & 57.7 (46.9-67.3) & - \\
\hline $71(6.1)$ & 6,632 (5.9) & - \\
\hline $32.0(25.4-40.4)$ & $30.0(29.3-30.8)$ & $\begin{array}{l}1.05(0.83-1.33) \\
0.85(0.68-1.09)^{b}\end{array}$ \\
\hline $59.3(51.1-69.4)$ & $60.6(52.3-69.0)$ & - \\
\hline $28(2.4)$ & $1,455(1.3)$ & - \\
\hline $12.4(8.5-17.9)$ & $6.5(6.1-6.8)$ & $1.76(1.18-2.62)$ \\
\hline 4 & 44 & - \\
\hline $1.8(0.7-4.7)$ & $0.2(0.1-0.3)$ & 8.69 (3.09-24.46) \\
\hline 24 & 1,411 & - \\
\hline $10.6(7.1-15.8)$ & $6.3(5.9-6.6)$ & $1.55(1.01-2.37)$ \\
\hline 9 & 267 & $0.96(0.49-1.87)$ \\
\hline 31 & 2,285 & $1.73(1.22-2.47)$ \\
\hline 64.9 (56.4-74.6) & $65.9(55.9-75.4)$ & - \\
\hline
\end{tabular}

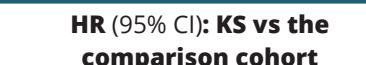

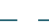

comparison cohort

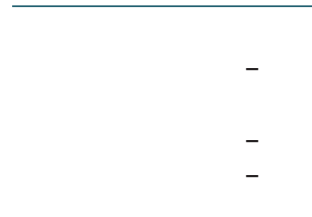

${ }^{a}$ KS: 33 DVT, 6 pulmonary embolisms. Comparison cohort: 632 DVT, 323 pulmonary embolisms, 7 central vein thromboses, and 11 portal vein thromboses. ${ }^{b}$ Adjusted model applying use of antidiabetics, antihypertensives, statins, platelet inhibitors, and anticoagulation therapy as time-varying covariates. 'KS: 25 myocardial infarctions, 42 stroke, and 13 transient ischaemic attacks. Comparison cohort: 3266 myocardial infarctions, 3323 strokes, and 807 transient ischaemic attacks.

$\mathrm{Cl}$, confidence interval; HR, hazard ratio; KS, Klinefelter syndrome.

A total of 9 men with KS and 153 men in the comparison cohort suffered both VTE and ATE.

In the KS cohort, $12.4 \quad(95 \%$ CI, 8.5-17.9) thrombotic deaths were recorded per 10,000 personyears, and in the comparison cohort 6.5 (6.1-6.8) thrombotic deaths were recorded per 10,000 personyears, giving an increased risk of thrombotic deaths among men with KS compared with the comparison cohort (Fig. 1C and Table 1). The increased risk of thrombotic death among men with KS was sustained, looking at deaths due to VTE or ATE separately (Table 1). The most common cause of death was pulmonary embolism and myocardial infarction, respectively. Post-thrombotic survival was not different when comparing men with KS and the comparison cohort regarding VTE, but all-cause mortality following any ATE was increased among men with KS compared with the comparison cohort (Table 1).

\section{Testosterone treatment in KS and effects on thrombotic outcomes}

Only $48.7 \%$ of men with KS redeemed prescriptions for testosterone during the study period (Table 2). Among the treated men with KS, 89 had prescription for transdermal testosterone only, 258 had prescription for injectable testosterone only, 174 had prescription for both transdermal and injectable testosterone, and 42 had prescription for oral testosterone only. Age at diagnosis of KS was not different when comparing those who have never received treatment and those receiving testosterone treatment, but those who have never received treatment were on average born 12 years earlier and diagnosed 15 years earlier (Fig. 2 and Table 2). Among those men with KS that never received testosterone treatment, biochemical analyses of testosterone and luteinizing hormone levels were available in 92 cases. https://ec.bioscientifica.com

https://doi.org/10.1530/EC-19-0433 (c) 2020 The authors Published by Bioscientifica Ltd

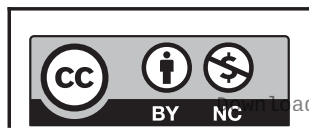

This work is licensed under a Creative Commons Attribution-NonCommercial 4.0 International License. ded from Bioscientifica.com at 04/26/2023 06:36:18AM via free access 
A

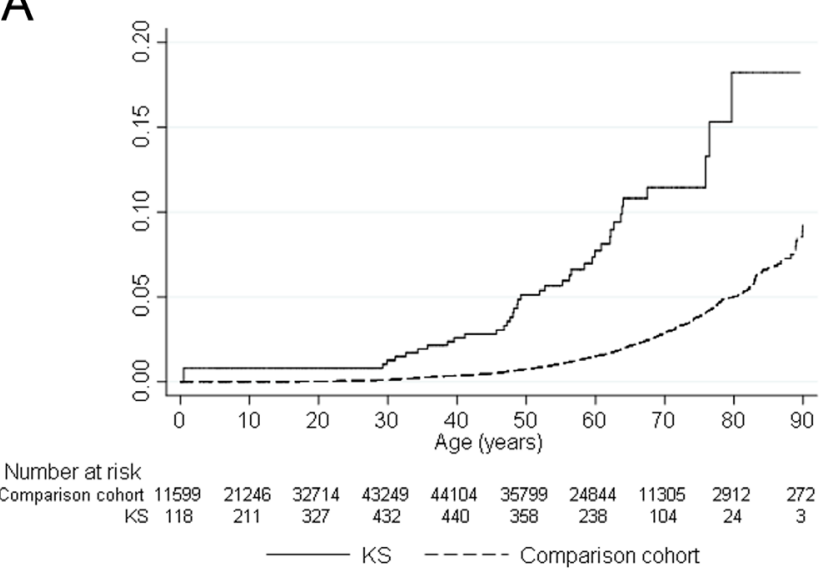

B

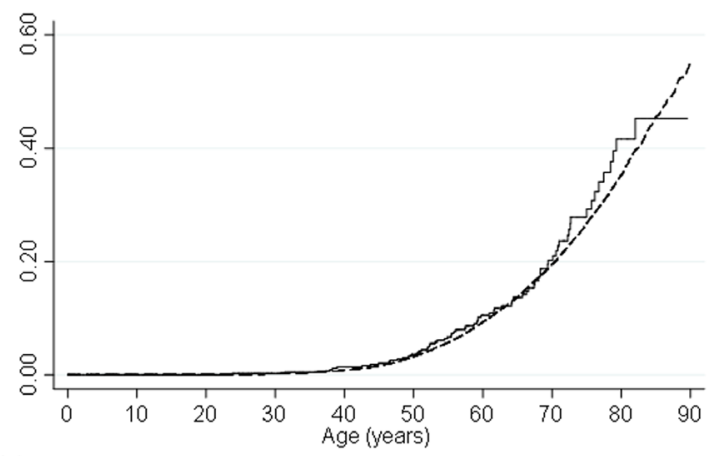

Number at risk

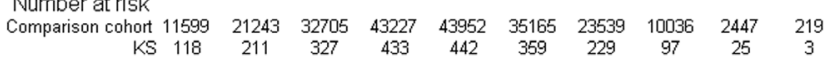
KS ----- Comparison cohort

C

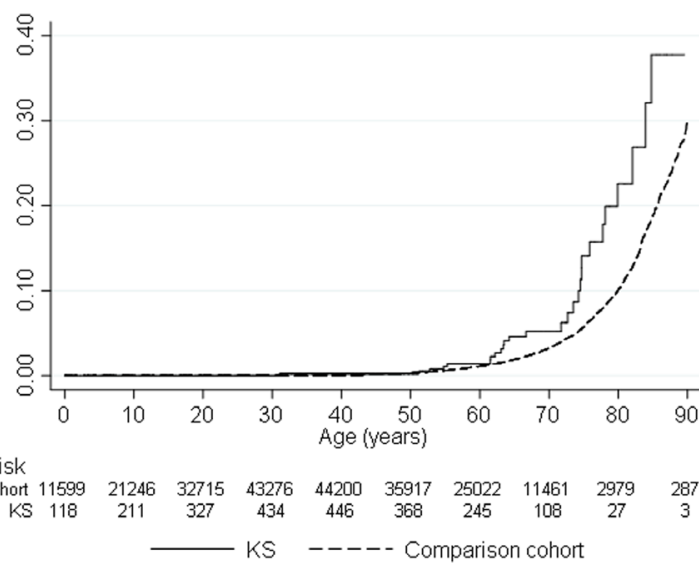

Figure 1

Thrombotic outcomes among participants. Kaplan-Meier plots. Venous thromboembolism (A), arterial thrombosis (B), and thrombotic deaths (C) KS, Klinefelter syndrome.

Of these, $72 \%(n=66)$ had biochemistry results indicative of hypogonadism. Of those with no clear biochemical evidence of hypogonadism, 35\% $(n=9)$ were less than 18 years and $38 \%(n=10)$ had a mosaic karyotype.
In our age-adjusted model, testosterone treatment was associated with a non-significant reduction in VTE events among men with KS (Fig. 3A and Table 2). In a model further adjusting for the use of medications indicative of co-morbidity, the HR was further lowered, but still insignificantly. The median time from first testosterone prescription to first VTE event was 7.0 years (full range, 1.2-22.2 years). Among men with KS, laboratory results were available within \pm 90 days of the VTE event in 19 cases $(50 \%)$ of which five where receiving testosterone treatment at the time of VTE. All presented with haematocrit and haemoglobin within normal range, and six of these men had available testosterone measurements with no one ever demonstrating concentrations above the normal range at any time during follow-up.

In the age-adjusted model, testosterone treatment was associated with a non-significant increase in ATE events among men with KS, but not after applying further adjustment for other prescription medications (Fig. 3B and Table 2). The median time from first testosterone prescription to first ATE event was 8.7 years (full range, 1.7-21.7 years). Among men with KS, laboratory results were available within \pm 90 days of the ATE event in 28 cases (39\%) of which 14 were receiving testosterone treatment at the time of ATE. One patient suffering myocardial infarction and one patient suffering transient ischemic attack presented with haematocrit and haemoglobin above the normal range. Of the 28 cases with available laboratory data \pm 90 days of the ATE event, 12 had testosterone measurements available at any time before or after the event. One case on testosterone treatment presented with an elevated total testosterone measured 139 days before suffering a stroke event, but haematocrit measured on the day of the event was within the normal range.

Among men with KS, testosterone treatment was associated with lower mortality due to thrombosis, although the association was non-significant (Table 2).

Testosterone-treated men with KS had a higher incidence of first prescriptions of medications indicative of co-morbidities and associated with thrombosis treatment or prophylaxis (Table 2).

Among testosterone-treated men with KS, the risk of VTE or ATE was not different between routes of testosterone administration ( $P>0.4$, Fisher's exact test).

\section{Medications and co-morbidity associated with thrombosis risk}

The use of anticoagulation, platelet inhibitors, statins, and antihypertensives was increased among men with

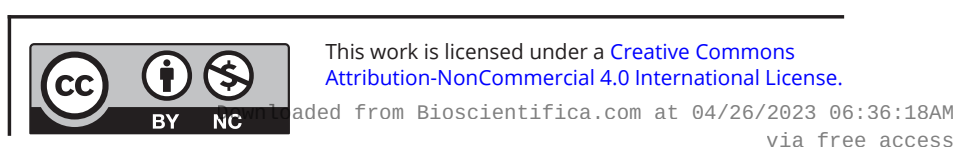


Table 2 Testosterone treatment and thrombotic outcomes in Klinefelter syndrome (KS).

\begin{tabular}{l}
\hline \\
\hline Ever testosterone treatment $(n(\%))$ \\
Person-years \\
Age at diagnosis (years, median (interquartile range)) \\
Year of diagnosis (median (interquartile range) \\
Birth year (median (interquartile range)) \\
Age at first testosterone prescription (years, median \\
(interquartile range)) \\
Thrombosis and thrombotic deaths \\
VTE ( $n$ ) \\
Events per 10,000 person-years (95\% CI) \\
Age at first VTE (years, median (interquartile range)) \\
ATE ( $n$ ) \\
Events per 10,000 person-years (95\% CI) \\
Age at first ATE (years, median (interquartile range)) \\
Thrombotic deaths $(n)$ \\
Events per 10,000 person-years (95\% CI) \\
VTE deaths $(n)$ \\
ATE deaths $(n)$ \\
Medication \\
Antidiabetics $(n)$ \\
Statins $(n)$ \\
Platelet inhibitors $(n)$ \\
Antihypertensives $(n)$
\end{tabular}

\begin{tabular}{c}
\hline Untreated KS \\
\hline $592(51.3)$ \\
16,221 \\
$27.6(18.2-34.1)$ \\
$1986(1975-2004)$ \\
$1961(1946-1977)$ \\
- \\
\\
29 \\
$18.1(12.6-26.1)$ \\
$49.2(38.6-62.1)$ \\
50 \\
$31.4(23.8-41.4)$ \\
$64.2(53.4-70.8)$ \\
25 \\
$15.4(10.4-22.8)$ \\
3 \\
22 \\
110 \\
167 \\
163 \\
322 \\
\end{tabular}

\begin{tabular}{c}
\hline Treated KS \\
\hline $563(48.7)$ \\
6,395 \\
$25.5(15.3-34.3)$ \\
$2001(1990-2009)$ \\
$1973(1962-1986)$ \\
$30.4(19.2-40.9)$ \\
\\
9 \\
$14.3(7.4-27.4)$ \\
$52.7(47.7-59.9)$ \\
21 \\
$33.6(21.9-51.6)$ \\
$52.4(47.7-61.0)$ \\
3 \\
$4.7(1.5-14.5)$ \\
$<3$ \\
$<3$ \\
57 \\
103 \\
61 \\
135 \\
\end{tabular}

\begin{tabular}{c}
\hline $\begin{array}{c}\text { HR }(95 \% \mathrm{Cl}): \\
\text { untreated } \mathbf{~ K S}^{\mathrm{a}}\end{array}$ \\
- \\
- \\
- \\
- \\
- \\
- \\
\\
- \\
$0.69(0.32-1.52)$ \\
$0.57(0.26-1.27)^{\mathrm{b}}$ \\
- \\
- \\
$1.42(0.83-2.42)$ \\
$1.12(0.66-1.92)^{\mathrm{b}}$ \\
- \\
- \\
$0.58(0.17-1.96)$ \\
$1.13(0.10-12.14)$ \\
$0.47(0.11-2.04)$ \\
$1.46(1.03-2.08)$ \\
$1.52(1.17-1.98)$ \\
$1.58(1.17-2.15)$ \\
$2.00(1.61-2.49)$
\end{tabular}

aTestosterone treatment as a time-varying covariate with adjustment for birth year. ${ }^{b}$ Adjusted for birth year and use of antidiabetics, antihypertensives, statins, platelet inhibitors, and anticoagulation therapy as time-varying covariates.

ATE, arterial thrombosis; Cl: confidence interval; HR, hazard ratio; VTE, venous thromboembolism.

KS compared with the comparison cohort, indirectly identifying co-morbidities associated with an increased thrombosis risk (Table 3). In particular, a HR (95\% CI) of 2.60 (2.20-3.09) was seen for antidiabetics comparing men with KS and the comparison cohort (Table 3). Diagnoses of hypertension, hypercholesterolemia, and in particular atrial arrhythmia, arteriosclerosis, angina

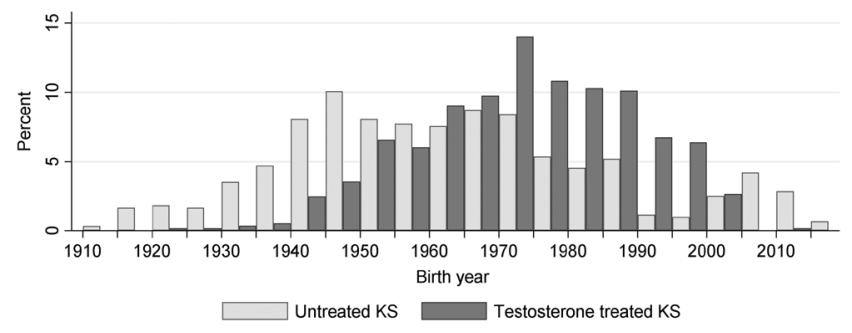

Figure 2

Distribution of birth year in untreated and treated Klinefelter syndrome (KS). In Denmark, testosterone treatment in KS is commonly not started until the time of expected puberty, explaining the apparent rise in numbers of untreated KS among those born after the year 2000. Also, the gradually lowering total number of KS seen after 1990 reflects the diagnostic lag in this condition. pectoris and diabetes, were more common among men with KS compared with the comparison cohort (Table 3).

Among all with VTE, $23.7 \%$ of men with KS and $9.2 \%$ in the comparison cohort had pre-existing diabetes $(P=0.008$, Fisher's exact test). No difference was seen between the groups for the incidence of hypertension, hypercholesterolemia, arteriosclerosis, angina pectoris or previous ATE as a predisposing VTE risk factor. A subsequent analysis comparing rates of VTE between KS and controls and including only those participants with diabetes yielded a HR (95\% CI) of 4.17 (1.08-16.03).

Predisposing ATE risk factors were all more common among men with KS compared with the comparison cohort, including diabetes (33.8\% vs $14.8 \%)$, hypertension $(71.8 \%$ vs $51.3 \%$ ), and previous VTE (7.0\% vs $1.0 \%$ ) ( $P \leq 0.001$ for all, Fisher's exact test). No difference was seen between the groups for the presence of hypercholesterolemia, arteriosclerosis or angina pectoris as a predisposing ATE risk factor. A subsequent analysis comparing rates of ATE between KS and controls and including only those participants with both diabetes and hypertension yielded a HR $(95 \%$ CI) of $0.79(0.45-1.40)$. 
A

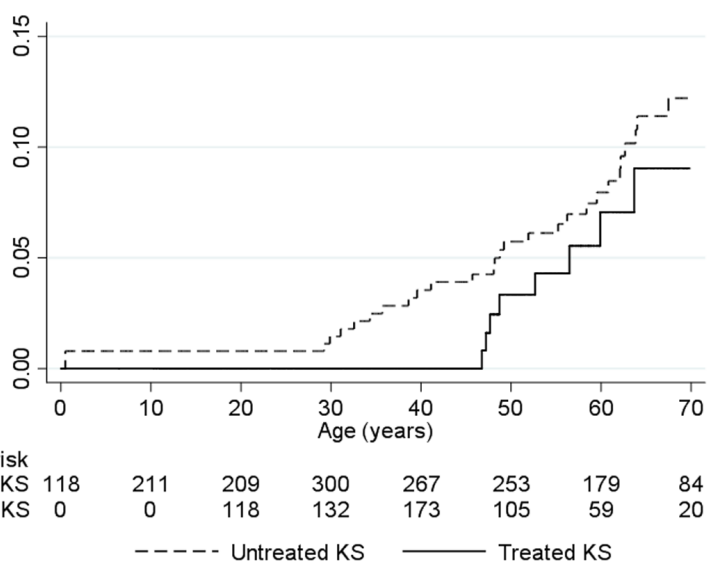

B

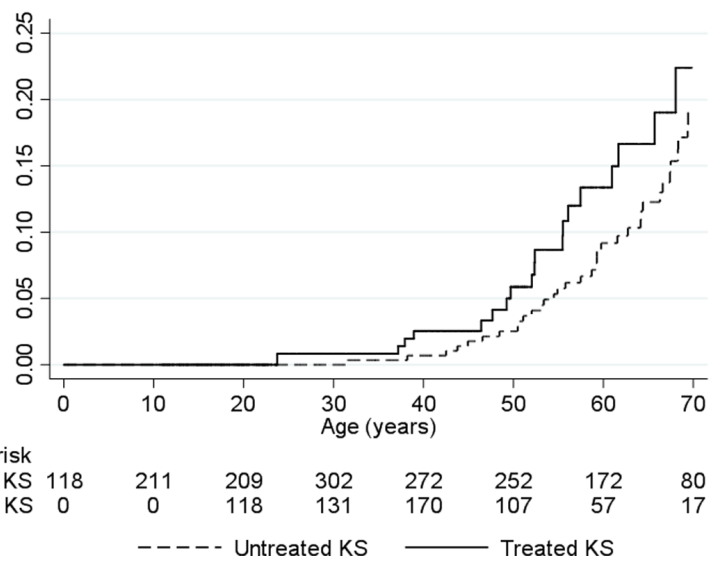

Figure 3

Thrombotic outcomes among testosterone-treated and -untreated Klinefelter syndrome (KS). Kaplan-Meier failure function for venous thromboembolism (A) and arterial thrombosis (B).

\section{Effect of 47,XXY karyotype vs mosaicism}

There was no difference in age at diagnosis or year of birth comparing 47,XXY and mosaic KS karyotypes (Supplementary Table 3). However, testosterone treatment was more frequent and initiated on average 13 years earlier in life in non-mosaic KS. Men with 47,XXY karyotype expressed an insignificantly lower rate of both VTE and ATE compared with mosaic karyotypes.

\section{Discussion}

Here, we present nationwide data from a large KS cohort, for the first time including information about use of testosterone and other prescription medicine allowing unique insight in the clinical care of men with KS.

This research show that the risk of thrombosis and thrombotic death is clearly elevated in KS, and that men with KS present with a pro-thrombotic phenotype characterized by a higher incidence of prothrombotic co-morbidities like diabetes, hypertension and hypercholesterolaemia and more prescriptions for medications associated with thrombosis prophylaxis. Additionally, men with KS suffering from thrombosis were more likely than men in the comparison cohort to present with underlying pro-thrombotic conditions. Although we could not formally show a lower thrombotic risk among testosterone treated men with KS, several lines of evidence pointed in that direction, including lower incidence rates for VTE and thrombotic deaths compared with the untreated KS cohort. Further, we speculate that the increased number of co-morbidity diagnoses and

Table 3 Medication and co-morbidities associated with thrombosis risk.

\begin{tabular}{l}
\hline \\
\hline Medication \\
Anticoagulation (\%) \\
Platelet inhibitors (\%) \\
Statins (\%) \\
Antihypertensives (\%) \\
Antidiabetics (\%) \\
Co-morbidity \\
Atrial arrhythmia (\%) \\
Hypertension (\%) \\
Diabetes (\%) \\
Hypercholesterolemia (\%) \\
Angina pectoris (\%) \\
Unstable angina (\%) \\
Arteriosclerosis
\end{tabular}

\begin{tabular}{c}
\hline KS \\
\hline 8.2 \\
19.3 \\
23.3 \\
39.5 \\
14.4 \\
8.2 \\
11.4 \\
11.9 \\
6.3 \\
8.6 \\
1.8 \\
1.12
\end{tabular}

\begin{tabular}{c}
\hline Comparison cohort \\
\hline 3.4 \\
13.8 \\
16.2 \\
28.6 \\
6.42 \\
4.0 \\
9.5 \\
4.9 \\
4.0 \\
5.8 \\
1.0 \\
0.11
\end{tabular}

HR $(95 \% \mathrm{Cl})$ : KS vs the comparison cohort

aDanish National Prescription Registry (1994-2016); b Danish National Patient Registry (1977-2016).

$\mathrm{Cl}$, confidence interval; $\mathrm{HR}$, hazard ratio; $\mathrm{KS}$, Klinefelter syndrome.

https://ec.bioscientifica.com

https://doi.org/10.1530/EC-19-0433 (c) 2020 The authors Published by Bioscientifica Ltd

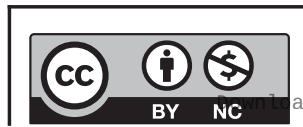

This work is licensed under a Creative Commons Attribution-NonCommercial 4.0 International License. ded from Bioscientifica.com at 04/26/2023 06:36:18AM via free access 
redeemed prescriptions among treated vs untreated KS, do not reflect a true difference in disease incidences, but rather could be representative of a higher standard of care in treated KS and missed diagnoses in the untreated KS group.

The current study adds new information about the influence of long term hypogonadism and testosterone treatment on thrombosis risk applying KS as a well-defined clinical disease model in a population-based nationwide matched cohort study with a 22-year follow-up time integrating diagnoses, medication use and clinical biochemistry. Importantly, we do not find evidence for any detrimental effects of testosterone treatment on incidences of cardiovascular complications. This is consistent with the findings of a recent cohort study with a 4-year follow-up assessing thrombotic complications following testosterone treatment in female-to-male transgender persons (28). In that study, adjusted rates of VTE or ATE were not different among 2118 testosteronetreated genetic females compared with reference men or women.

Men with KS on testosterone treatment presenting with thrombosis did not commonly present with erythrocytosis based on available biochemical evidence, and the duration from first testosterone prescription to any thrombotic events was more than 1.2 years. This indicates that testosterone treatment in KS was not associated with a 'starter-effect' influencing VTE risk. This is in contrast with the findings of a national British case-control study among an unselected group of men receiving testosterone treatment (5), but further implies that testosterone treatment in KS is safe. A side effect to testosterone treatment mentioned as a safety warning by the FDA is VTE, but in the setting of KS and hypogonadism, it seems that any unwarranted effects of testosterone treatment on VTE risk is balanced out and even overpowered by beneficial effects of hormone supplementation. Testosterone treatment could act to alleviate VTE risk in Klinefelter syndrome by reversing the vicious cycle of hypogonadism-induced obesity and insulin insensitivity.

Death due to ATE was increased in the KS cohort compared with the comparison cohort, although the incidence of non-fatal ATE was not different. The latter is somewhat surprising considering the excess of cardiovascular risk factors among men with KS. Speculatively, an explanation could be that the genetic composition of KS with two $\mathrm{X}$ chromosomes, similar to what is seen in women in comparison to men (29), could be affecting the clinical presentation of ATE in the patients, and that males with KS are actually protected from ATE, much as pre-menopausal women have a lower ATE risk than age-matched males (30). Also, the increased rates of secondary prophylactic medications such as statins and platelet inhibitors in men with KS compared with the comparison cohort could affect ATE risk comparison between the groups, although adjusting for use of said medications did not alter the estimates significantly. The increased all-cause mortality following ATE among men with KS compared with the comparison cohort, could indicate that the increased co-morbidity load as evidenced in this cohort, added to the composite phenotype of KS with a poorer socioeconomic profile (31, 32 ), is affecting the capability for rehabilitation after ATE.

We present the first large-scale assessment of testosterone use among men with KS and rather discouragingly found that only about half of all men with KS were offered testosterone treatment. The untreated KS population consisted of two groups, a small group of younger individuals who at the end of the study period had not yet reached pubertal age, and a larger group of men with KS that were on average born 12 years before the testosterone-treated men with KS. We speculate that the finding is indicative of how the attitude towards KS has improved in the Danish health care system over time, with more patients in later years receiving appropriate care in specialized clinics, but also that the older KS population is not being offered optimal treatment. Knowing that hypergonadotropic hypogonadism is virtually omnipresent among KS, and although non-treatment could be by choice, we show that the majority of men with KS not being offered testosterone treatment indeed had laboratory findings suggestive of hypogonadism indicating a lapse in clinical care. There is a need to further explore the traits of those men with KS-treated vs the non-treated to elaborate on the reasons for non-treatment in an unexpectedly high proportion of the Danish KS population.

We found that the incidence of VTE was still increased among men with KS when restricting the analysis to only include participants with diabetes. This could indicate that genome wide modifications induced by the presence of a supernumerary X-chromosome $(33,34)$ could also be contributing directly to thrombosis risk in KS, affecting the external validity of our findings which might not apply to all groups of males with hypogonadism.

The strength of this national study is the use of KS diagnoses verified by karyotyping and applying a large age matched comparison cohort and having a long follow-up time. We utilized data from several high-quality

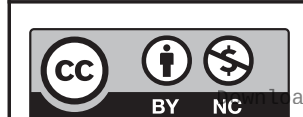


registries $(19,20,22)$, applying a verified validation method for VTE events (24). The study is limited by potential misclassification due to non-diagnosis of KS in the comparison cohort. However, this would attenuate the true association and cannot explain the excess of VTE events observed among men with known KS. Also, thrombotic events and deaths occurring prior to the beginning of the study period were not assessed, potentially introducing bias by positively selection of survivors. However, the mean age at entry into the study was just over 25 years minimizing the risk of having missed any events leading up to entry in the study. Differential outcome detection rates between $\mathrm{KS}$ and the comparison cohort could also be affecting our estimates. Men with KS could be more inclined to seek medical attention compared with men in the comparison cohort, increasing the likelihood of appointment of additional diagnoses. However, our data if anything point to a lapse of care in men with KS, with many males not receiving appropriate testosterone treatment, complicating conclusions on the risk of surveillance bias. Lastly, the comparison between men with KS and men in the background population is sensitive to the presence of unmeasured bias, as for instance weight or social status is not recorded in the applied registries. However, we know that living with KS has consequences traversing all aspects of life (1), and any such differences between the cohorts could then be seen as an intrinsic part of the syndrome.

The data quality of the registries is generally high, but we cannot rule out that thrombotic events diagnosed within the primary care system might not have been recognized. Also the Danish National Prescription Registry only records redeemed prescriptions, and misclassification of the true intake of testosterone and other medications cannot be ruled out, as also illicit use of androgenic compounds cannot be assessed.

In some aspects our analyses might suffer from being insufficiently powered to draw firm conclusions, especially regarding the clinical consequences of testosterone treatment in men with KS. Our intention, however, was to present the best available data, and we do feel confident that the data at hand is strong enough to exclude a potentially overlooked critical impact of testosterone treatment in $\mathrm{KS}$ on thrombotic risk, reassuring us that this type of treatment appears safe.

In conclusion, men with $\mathrm{KS}$ expressed a more prothrombotic morbidity pattern and higher rates of VTE and death due to thrombosis. Testosterone treatment in KS was not associated with an increased risk of thrombosis. Only about half of the KS population was treated with testosterone, highlighting the need for a clinical guideline for treatment of men with KS and to stress the implementation of specialized clinics.

\section{Supplementary materials}

This is linked to the online version of the paper at https://doi.org/10.1530/ EC-19-0433.

\section{Declaration of interest}

Claus Gravholt is a Senior Editor for Endocrine Connections. Claus was not involved in the review or editorial process for this paper, on which he is listed as an author. The other authors have nothing to disclose.

\section{Funding}

This work was supported by the University of Southern Denmark (grant number: N/A), The Region of Southern Denmark (grant number (N/A), the Lundbeck Foundation (grant number (N/A), and Novo Nordisk Foundation (grant agreement NNF130C0003234 and NNF150C0016474).

\section{References}

1 Gravholt $\mathrm{CH}$, Chang S, Wallentin M, Fedder J, Moore P \& Skakkebaek A. Klinefelter syndrome - integrating genetics, neuropsychology and endocrinology. Endocrine Reviews 201839 389-423. (https://doi.org/10.1210/er.2017-00212)

2 Kloner RA, Carson 3rd C, Dobs A, Kopecky S \& Mohler 3rd ER. Testosterone and cardiovascular disease. Journal of the American College of Cardiology 201667 545-557. (https://doi.org/10.1016/j. jacc.2015.12.005)

3 Morgentaler A, Miner MM, Caliber M, Guay AT, Khera M \& Traish AM. Testosterone therapy and cardiovascular risk: advances and controversies. Mayo Clinic Proceedings 201590 224-251. (https:// doi.org/10.1016/j.mayocp.2014.10.011)

4 Ruige JB, Ouwens DM \& Kaufman JM. Beneficial and adverse effects of testosterone on the cardiovascular system in men. Journal of Clinical Endocrinology and Metabolism 201398 4300-4310. (https:// doi.org/10.1210/jc.2013-1970)

5 Martinez C, Suissa S, Rietbrock S, Katholing A, Freedman B, Cohen AT \& Handelsman DJ. Testosterone treatment and risk of venous thromboembolism: population based case-control study. BMJ 2016355 i5968. (https://doi.org/10.1136/bmj.i5968)

6 Vigen R, O’Donnell CI, Baron AE, Grunwald GK, Maddox TM, Bradley SM, Barqawi A, Woning G, Wierman ME, Plomondon ME, et al. Association of testosterone therapy with mortality, myocardial infarction, and stroke in men with low testosterone levels. JAMA 2013310 1829-1836. (https://doi.org/10.1001/jama.2013.280386)

7 Basaria S, Coviello AD, Travison TG, Storer TW, Farwell WR, Jette AM, Eder R, Tennstedt S, Ulloor J, Zhang A, et al. Adverse events associated with testosterone administration. New England Journal of Medicine 2010363 109-122. (https://doi.org/10.1056/ NEJMoa1000485)

8 Bojesen A, Juul S, Birkebaek NH \& Gravholt CH. Morbidity in Klinefelter syndrome: a Danish register study based on hospital discharge diagnoses. Journal of Clinical Endocrinology and Metabolism 200691 1254-1260. (https://doi.org/10.1210/jc.2005-0697)

9 Zoller B, Ji J, Sundquist J \& Sundquist K. High risk of venous thromboembolism in Klinefelter syndrome. Journal of the American Heart Association 20165 e003567. (https://doi.org/10.1161/ JAHA.116.003567) 
10 Bojesen A \& Gravholt CH. Morbidity and mortality in Klinefelter syndrome (47,XXY). Acta Paediatrica 2011100 807-813. (https://doi. org/10.1111/j.1651-2227.2011.02274.x)

11 Bojesen A, Juul S, Birkebaek N \& Gravholt CH. Increased mortality in Klinefelter syndrome. Journal of Clinical Endocrinology and Metabolism 200489 3830-3834. (https://doi.org/10.1210/jc.2004-0777)

12 Swerdlow AJ, Higgins CD, Schoemaker MJ, Wright AF, Jacobs PA \& United Kingdom Clinical Cytogenetics Group. Mortality in patients with Klinefelter syndrome in Britain: a cohort study. Journal of Clinical Endocrinology and Metabolism 200590 6516-6522. (https:// doi.org/10.1210/jc.2005-1077)

13 Carrageta DF, Oliveira PF, Alves MG \& Monteiro MP. Obesity and male hypogonadism: tales of a vicious cycle. Obesity Reviews 201920 1148-1158. (https://doi.org/10.1111/obr.12863)

14 Chang S, Biltoft D, Skakkebaek A, Fedder J, Bojesen A, Bor MV, Gravholt $\mathrm{CH} \&$ Munster $\mathrm{AB}$. Testosterone treatment and association with thrombin generation and coagulation inhibition in Klinefelter syndrome: a cross-sectional study. Thrombosis Research 2019182 175-181. (https://doi.org/10.1016/j.thromres.2019.08.011)

15 Di Minno MN, Esposito D, Di Minno A, Accardo G, Lupoli G, Cittadini A, Giugliano D \& Pasquali D. Increased platelet reactivity in Klinefelter men: something new to consider. Andrology 20153 876-881. (https://doi.org/10.1111/andr.12080)

16 Bojesen A, Host C \& Gravholt $\mathrm{CH}$. Klinefelter's syndrome, type 2 diabetes and the metabolic syndrome: the impact of body composition. Molecular Human Reproduction 201016 396-401. (https://doi.org/10.1093/molehr/gaq016)

17 Lippi G, Franchini M \& Favaloro EJ. Thrombotic complications of erythropoiesis-stimulating agents. Seminars in Thrombosis and Hemostasis 201036 537-549. (https://doi. org/10.1055/s-0030-1255448)

18 Byrnes JR \& Wolberg AS. Red blood cells in thrombosis. Blood 2017 130 1795-1799. (https://doi.org/10.1182/blood-2017-03-745349)

19 Schmidt M, Pedersen L \& Sorensen HT. The Danish Civil Registration System as a tool in epidemiology. European Journal of Epidemiology 201429 541-549. (https://doi.org/10.1007/s10654-014-9930-3)

20 Schmidt M, Schmidt SA, Sandegaard JL, Ehrenstein V, Pedersen L, Sorensen HT. The Danish National Patient Registry: a review of content, data quality, and research potential. Clinical Epidemiology 20157 449-490. (https://doi.org/10.2147/CLEP.S91125)

21 Helweg-Larsen K. The Danish register of causes of death. Scandinavian Journal of Public Health 201139 26-29. (https://doi. org/10.1177/1403494811399958)

22 Kildemoes HW, Sorensen HT, Hallas J. The Danish National Prescription Registry. Scandinavian Journal of Public Health 201139 38-41. (https://doi.org/10.1177/1403494810394717)

23 Videbech P \& Nielsen J. Electronic data processing in the Danish cytogenetic central register and EDP problems of registers in general. Clinical Genetics 197915 137-146. (https://doi. org/10.1111/j.1399-0004.1979.tb01752.x)
24 Schmidt M, Cannegieter SC, Johannesdottir SA, Dekkers OM, Horvath-Puho E \& Sorensen HT. Statin use and venous thromboembolism recurrence: a combined nationwide cohort and nested case-control study. Journal of Thrombosis and Haemostasis 201412 1207-1215. (https://doi.org/10.1111/jth.12604)

25 Sundboll J, Adelborg K, Munch T, Froslev T, Sorensen HT, Botker HE $\&$ Schmidt M. Positive predictive value of cardiovascular diagnoses in the Danish National Patient Registry: a validation study. BMJ Open 20166 e012832. (https://doi.org/10.1136/bmjopen-2016-012832)

26 Anand SS, Islam S, Rosengren A, Franzosi MG, Steyn K, Yusufali AH, Keltai M, Diaz R, Rangarajan S, Yusuf S, et al. Risk factors for myocardial infarction in women and men: insights from the INTERHEART study. European Heart Journal 200829 932-940. (https://doi.org/10.1093/eurheartj/ehn018)

27 Stubbs MJ, Mouyis M \& Thomas M. Deep vein thrombosis. BMJ 2018 360 k351. (https://doi.org/10.1136/bmj.k351)

28 Getahun D, Nash R, Flanders WD, Baird TC, Becerra-Culqui TA, Cromwell L, Hunkeler E, Lash TL, Millman A, Quinn VP, et al. Cross-sex hormones and acute cardiovascular events in transgender persons: a cohort study. Annals of Internal Medicine 2018169 205-213. (https://doi.org/10.7326/M17-2785)

29 Mehta LS, Beckie TM, DeVon HA, Grines CL, Krumholz HM, Johnson MN, Lindley KJ, Vaccarino V, Wang TY, Watson KE, et al. Acute myocardial infarction in women: a scientific statement from the American Heart Association. Circulation 2016133 916-947. (https://doi.org/10.1161/CIR.0000000000000351)

30 Leening MJ, Ferket BS, Steyerberg EW, Kavousi M, Deckers JW, Nieboer D, Heeringa J, Portegies ML, Hofman A, Ikram MA, et al. Sex differences in lifetime risk and first manifestation of cardiovascular disease: prospective population based cohort study. BMJ 2014349 g5992. (https://doi.org/10.1136/bmj.g5992)

31 Bojesen A, Stochholm K, Juul S \& Gravholt CH. Socioeconomic trajectories affect mortality in Klinefelter syndrome. Journal of Clinical Endocrinology and Metabolism 201196 2098-2104. (https:// doi.org/10.1210/jc.2011-0367)

32 Stochholm K, Bojesen A, Jensen AS, Juul S \& Gravholt CH. Criminality in men with Klinefelter's syndrome and XYY syndrome: a cohort study. BMJ Open 20122 e000650. (https://doi.org/10.1136/ bmjopen-2011-000650)

33 Sharma A, Jamil MA, Nuesgen N, Schreiner F, Priebe L, Hoffmann P, Herns S, Nothen MM, Frohlich H, Oldenburg J, et al. DNA methylation signature in peripheral blood reveals distinct characteristics of human X chromosome numerical aberrations. Clinical Epigenetics 20157 76. (https://doi.org/10.1186/s13148-0150112-2)

34 Skakkebæk A, Nielsen MM, Trolle C, Vang S, Hornshøj H, Hedegaard J, Wallentin M, Bojesen A, Hertz JM, Fedder J, et al. DNA hypermethylation and differential gene expression associated with Klinefelter syndrome. Scientific Reports 20188 13740. (https://doi. org/10.1038/s41598-018-31780-0)

Received in final form 9 December 2019

Accepted 11 December 2019

Accepted Manuscript published online 11 December 2019 https://ec.bioscientifica.com https://doi.org/10.1530/EC-19-0433 (c) 2020 The authors Published by Bioscientifica Ltd

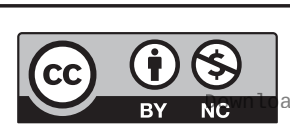

This work is licensed under a Creative Commons Attribution-NonCommercial 4.0 International License. ded from Bioscientifica, com at 04/26/2023 06:36:18AM 